\title{
Video-assisted thoracic surgery does not reduce the incidence of postoperative atrial fibrillation after pulmonary lobectomy
}

\author{
Bernard J. Park, MD, ${ }^{a}$ Hao Zhang, MD, ${ }^{\text {b }}$ Valerie W. Rusch, MD, ${ }^{a}$ and David Amar, $\mathrm{MD}^{\mathrm{b}}$
}

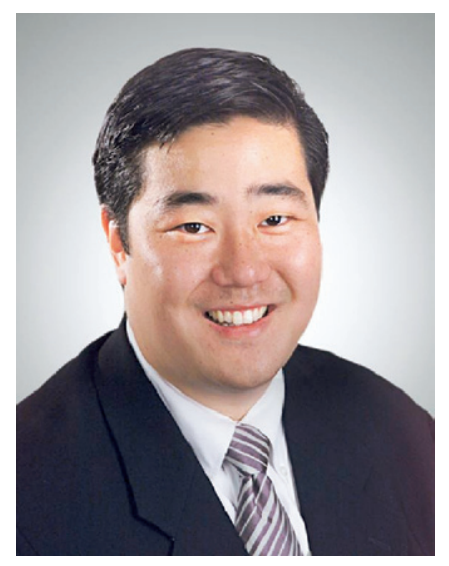

Dr Park

From the Departments of Surgery ${ }^{\mathrm{a}}$ and Anesthesiology and Critical Care Medicine, ${ }^{\mathrm{b}}$ Memorial Sloan-Kettering Cancer Center, New York, NY.

Received for publication July 18, 2006; revisions received Sept 19, 2006; accepted for publication Sept 29, 2006.

Reprint requests: Bernard J. Park, MD, Thoracic Service, Department of Surgery, Memorial Sloan-Kettering Cancer Center, 1275 York Avenue, Room C-867, New York, NY 10021 (E-mail: parkb@mskcc.org).

J Thorac Cardiovasc Surg 2007;133:775-9

0022-5223/ $\$ 32.00$

Copyright (C) 2007 by The American Association for Thoracic Surgery

doi:10.1016/j.jtcvs.2006.09.022
Objective: The objective was to define the incidence of atrial fibrillation after video-assisted thoracic surgery lobectomy and determine whether video-assisted thoracic surgery reduces atrial fibrillation rate compared with thoracotomy.

Methods: With the use of a single-institution database of patients who underwent lobectomy for clinical stage I non-small cell lung cancer, 389 patients were identified who were in sinus rhythm preoperatively and received no prophylactic antiarrhythmics. Patients undergoing video-assisted thoracic surgery were age and gender matched with those undergoing thoracotomy.

Results: After matching, 122 patients undergoing video-assisted thoracic surgery and 122 patients undergoing thoracotomy were eligible for analysis. Patients undergoing video-assisted thoracic surgery had a higher preoperative diffusion capacity $(92 \% \pm 28 \%$ vs $80 \% \pm 18 \%$ predicted, $P=.001)$ and a lower rate of induction chemotherapy $(5 / 122,4 \%$ vs $11 / 122,11 \%, P=.05)$ than patients undergoing thoracotomy. Atrial fibrillation occurred in $12 \%$ of patients $(15 / 122)$ undergoing video-assisted thoracic surgery and $16 \%$ of patients (20/122) undergoing thoracotomy $(P=.36)$. Overall, complications were lower in the video-assisted thoracic surgery group $(17.2 \%$ vs $27.9 \%, P=.046)$. Patients with atrial fibrillation were older in both video-assisted thoracic surgery $(73 \pm 7$ years vs $66 \pm 9$ years, $P=$ $.002)$ and thoracotomy groups ( $72 \pm 7$ years vs $66 \pm 10$ years, $P=.005)$. Length of stay for patients with atrial fibrillation was greater in both video-assisted thoracic surgery $(6.0 \pm 1.5$ days vs $4.7 \pm 2.5$ days, $P=.01)$ and thoracotomy groups $(9.2$ \pm 4.3 days vs $6.8 \pm 3.6$ days, $P=.03$ ).

Conclusions: Regardless of surgical approach, atrial fibrillation after lobectomy occurred with equal frequency. This supports the theory that autonomic denervation and stress-mediated neurohumoral mechanisms are responsible for the pathogenesis of postoperative atrial fibrillation. Prophylaxis regimens against atrial fibrillation should be the same for either operative approach.

$\mathrm{P}$ ostoperative atrial arrhythmias, in particular atrial fibrillation (AF), are seen in more than $20 \%$ of elderly patients undergoing noncardiac thoracic surgery. ${ }^{1}$ Postoperative AF is commonly associated with other complications, resulting in increased hospital stay and costs, as well as greater risk of stroke in those who remain in persistent AF. ${ }^{1,2}$ Advanced age ( $\geq 60$ years) is the independent risk factor most strongly and consistently associated with postoperative $\mathrm{AF} \cdot{ }^{1-3}$ Fifty percent of patients with non-small cell lung cancer (NSCLC) are aged more than 65 years, whereas more than $30 \%$ are at least 70 years old at diagnosis. ${ }^{4,5}$ This, combined with the fact that the proportion of individuals aged more than 65 years in the United States is increasing, suggests that the number of elderly patients with lung cancer requiring major thoracic surgical procedures will continue to increase in the future. ${ }^{6}$ 


\section{Abbreviations and Acronyms \\ $\mathrm{AF}=$ atrial fibrillation \\ NSCLC $=$ non-small cell lung cancer \\ VATS $=$ video-assisted thoracic surgery}

The technique of video-assisted thoracic surgery (VATS) pulmonary lobectomy for NSCLC was first reported in the early 1990s simultaneously by several authors. ${ }^{7-10}$ When used with a non-rib spreading technique, a VATS approach has been shown to be associated with a shorter hospital stay and decreased acute postoperative pain. ${ }^{10,11}$ Because of this and indications that the procedure is safe and oncologically acceptable in patients with clinical stage I disease, the use of VATS lobectomy for primary surgical therapy of earlystage NSCLC has been slowly increasing. ${ }^{12-17}$ There is also a belief that the use of a minimally invasive VATS technique may result in superior rates of postoperative morbidity when compared with thoracotomy, especially in elderly or high-risk populations. ${ }^{18,19}$

However, relatively few studies have analyzed in detail the incidence of postoperative AF after VATS lobectomy or whether this rate is decreased in these patients when compared with those undergoing thoracotomy. We decided to perform a case-control study analyzing the rates of postoperative arrhythmia in patients undergoing lobectomy to determine whether there is an advantage afforded by the minimally invasive VATS approach.

\section{Patients and Methods}

After approval by the institutional review board and waiver of informed consent, a prospectively maintained database of all patients undergoing thoracic surgery for lung cancer at a single institution was used to identify all patients who underwent elective pulmonary lobectomy for clinical stage I NSCLC and were in sinus rhythm preoperatively. Excluded were patients who were not in sinus rhythm before surgery, taking class I or III antiarrhythmic drugs, or placed on prophylactic medications postoperatively. Individuals already taking preoperative beta-blockers or calcium channel blockers for hypertension or coronary artery disease were resumed on the first postoperative day to avoid withdrawal and were not excluded. Also excluded were patients who underwent a lesser resection (exploration, wedge or segmentectomy) or more extensive operation (bilobectomy, pneumonectomy, chest wall resection, or major vascular resection).

Standard anesthesia induction and maintenance regimens and intraoperative fluid restriction were used for all patients. Postoperative pain relief was provided by continuous administration of epidural opioid (usually fentanyl with bupivacaine $0.05 \%$ ) administration. In all patients, anatomic pulmonary lobectomy and ipsilateral mediastinal lymph node dissection were performed either by VATS or standard posterolateral thoracotomy. VATS lobectomy was defined as anatomic pulmonary lobectomy using a video thoracoscope and 3 non-rib spreading incisions, the largest of which was a 3- to 4-cm utility incision. Patients converted from VATS for whatever reason were considered to be in the thoracotomy group. All patients remained overnight in the postanesthesia care unit on continuous telemetry and were then discharged to a dedicated thoracic surgical ward on the first postoperative day. When available, continuous telemetry was continued on the ward for an additional 48 hours or longer as needed. If the patient remained in normal sinus rhythm for 72 hours postoperatively, telemetry was discontinued. Data on patient characteristics, operative details, and postoperative recovery were collected in a prospective database approved by the institutional review board and analyzed retrospectively. All complications were graded according to the National Cancer Institute Common Terminology Criteria for Adverse Events version 3.0 (http://ctep.cancer.gov/reporting/ ctc.html). AF was defined by an irregular rhythm with absent $\mathrm{P}$ waves confirmed by 12-lead electrocardiography. Episodes of AF typically were identified by continuous telemetry or 12-lead electrocardiography performed because of clinically detected tachycardia. Patients were considered to have AF if the episode lasted more than 5 minutes by continuous telemetry or required intervention because of rapid ventricular response, presence of symptoms, or hemodynamic compromise.

Patients were matched on the basis of age and gender. Differences between the VATS and thoracotomy groups were assessed by the Student $t$, chi-square, or Fisher exact tests where appropriate. Data are presented as mean value \pm standard deviation unless otherwise indicated. Statistical analysis was performed with the Statistical Package for the Social Sciences version 12.0 (SPSS Inc, Chicago, Ill).

\section{Results}

\section{Patient Characteristics and Atrial Fibrillation Rate}

A review of the prospective database of thoracic surgical cases performed from January 1999 to June 2004 identified 389 patients who were in sinus rhythm preoperatively, underwent elective lobectomy, and received no prophylactic antiarrhythmic medications. Of these patients, 267 underwent thoracotomy and 122 had a VATS approach. After age and gender matching, there were 244 patients eligible for analysis, 122 in each group. The patients in each group were well matched with respect to preoperative characteristics (Table 1). The VATS group had a slightly higher preoperative carbon monoxide diffusion in the lung, and a higher percentage of patients in the thoracotomy group received preoperative chemotherapy. The presence of comorbid conditions, preoperative serum electrolyte values, and the proportion of patients taking beta-blockers or calcium channel blockers in each group were similar. The operative time was slightly longer in the VATS group $(3.7 \pm 1.0$ hours vs 3.0 \pm 1.0 hours, $P=.001$ ).

Because of limited availability, continuous telemetry on transfer from the postanesthesia care unit was used in $23 \%$ of patients (57/244), with no difference between groups (25\% VATS vs $22 \%$ thoracotomy, $P=.76$ ). The overall rate of postoperative AF in all patients was $14 \%(35 / 244)$, and there was no difference in rates according to the surgi- 
TABLE 1. Patient characteristics

\begin{tabular}{|c|c|c|c|}
\hline Characteristics & $\begin{array}{c}\text { VATS } \\
\text { (n= 122) }\end{array}$ & $\begin{array}{c}\text { THOR } \\
\text { (n= 122) }\end{array}$ & $\begin{array}{c}P \\
\text { value }\end{array}$ \\
\hline Age, y & $67 \pm 10$ & $67 \pm 10$ & .99 \\
\hline Range, y & $38-85$ & $37-85$ & \\
\hline Male/female, $\mathrm{n}$ & $38 / 84$ & $38 / 84$ & .99 \\
\hline $\begin{array}{l}\text { Preoperative chemotherapy, } \mathrm{n} \\
\quad(\%)\end{array}$ & $5(4)$ & $13(11)$ & .05 \\
\hline COPD, n (\%) & $25(21)$ & $33(27)$ & .23 \\
\hline HTN, n (\%) & $46(38)$ & $40(33)$ & .42 \\
\hline $\mathrm{MI}, \mathrm{n}(\%)$ & $4(3)$ & $4(3)$ & .99 \\
\hline$C A D, n(\%)$ & $12(9.8)$ & $11(9.0)$ & 1.0 \\
\hline $\mathrm{DM}, \mathrm{n}(\%)$ & $10(8)$ & $9(7)$ & .81 \\
\hline $\mathrm{FEV}_{1} \%$ predicted & $90 \pm 19$ & $86 \pm 14$ & .09 \\
\hline DLCO \% predicted & $92 \pm 28$ & $80 \pm 18$ & .001 \\
\hline $\begin{array}{l}\text { Preoperative serum potassium } \\
\text { (mEq/L) }\end{array}$ & $4.3 \pm 0.4$ & $4.4 \pm 0.4$ & .49 \\
\hline $\begin{array}{l}\text { Preoperative serum calcium } \\
\text { (mg/dL) }\end{array}$ & $9.3 \pm 0.5$ & $9.3 \pm 0.6$ & .94 \\
\hline Preoperative $\beta$-blocker, $\mathrm{n}(\%)$ & $25(20)$ & $26(21)$ & 1.0 \\
\hline $\begin{array}{l}\text { Preoperative } \mathrm{Ca}^{2+} \text { channel } \\
\text { blocker, } \mathrm{n}(\%)\end{array}$ & $14(12)$ & $16(13)$ & .85 \\
\hline Duration of surgery (h) & $3.7 \pm 1.0$ & $3.0 \pm 1.0$ & .001 \\
\hline Telemetry, n (\%) & $30(25)$ & $27(22)$ & .76 \\
\hline
\end{tabular}

COPD, Chronic obstructive pulmonary disease; HTN, hypertension; MI, myocardial infarction; $C A D$, coronary artery disease; $D M$, diabetes mellitus; $F E V_{1}$, forced expiratory volume in 1 second; DLCO, carbon monoxide diffusion in the lung; VATS, video-assisted thoracic surgery; THOR, thoracotomy. Data are mean \pm standard deviation or $\mathrm{n}(\%)$.

cal approach (Table 2). AF was observed in 20 of 122 patients $(16 \%)$ who underwent thoracotomy and lobectomy compared with 15 of 122 patients (12\%) who underwent VATS lobectomy $(P=.36)$. Among patients who were 60 years and older, AF occurred in 15 of $100(15 \%)$ in the VATS group and in 18 of $100(18 \%)$ in the thoracotomy group $(P=.57)$. A similar proportion of patients on telemetry in the VATS and thoracotomy groups had AF (27\% vs

TABLE 2. Postoperative complications

\begin{tabular}{lccc}
\hline Feature & $\begin{array}{c}\text { VATS } \\
\text { (n= 122) }\end{array}$ & $\begin{array}{c}\text { THOR } \\
\text { (n= 122) }\end{array}$ & $\boldsymbol{P}$ value \\
\hline AF rate (\%) & $15 / 122(12 \%)$ & $20 / 122(16 \%)$ & .36 \\
Atelectasis, n & $2(1.6 \%)$ & $5(4.1 \%)$ & .28 \\
Prolonged air leak, n & $4(3.8 \%)$ & $7(5.7 \%)$ & .54 \\
Pneumothorax, n & $1(0.8 \%)$ & $3(2.5 \%)$ & .62 \\
Pneumonitis, n & $2(1.6 \%)$ & $5(4.1 \%)$ & .28 \\
Total, n (\%) & $21(17.2)$ & $34(27.9)$ & .046 \\
LOS, d & $4.9 \pm 2.4$ & $7.2 \pm 3.8$ & .001 \\
Deaths* ${ }^{*}, \mathrm{n}(\%)$ & $0(0 \%)$ & $3(2.5 \%)$ & .25 \\
\hline AF, Atrial & &
\end{tabular}

$A F$, Atrial fibrillation/flutter; LOS, length of stay; VATS, video-assisted thoracic surgery; THOR, thoracotomy. *Within 30 days of surgery. Total complication rate includes patients who had 1 or more events. Data are mean \pm standard deviation or $\mathrm{n}(\%)$.
$33 \%$ ). In addition, there were no differences in the presence of comorbid conditions, the preoperative serum electrolyte values, the proportion of patients taking beta-blockers or calcium channel blockers, or the operative time between patients who did and did not have AF (data not shown). Nine of the patients with AF had another complication as well.

\section{Perioperative Morbidity and Outcomes}

A total of 55 of 244 patients (23\%) had 1 or more postoperative complications, with a significantly lower complication rate in the VATS group (17.2\% in the VATS group vs $27.9 \%$ in the thoracotomy group, $P=.046)$. There was no difference in the perioperative 30-day mortality rates between the 2 groups, although there were no deaths in the VATS group. The mean length of hospital stay of the patients who underwent thoracotomy was more than 2 days greater than that of the patients who underwent VATS (7.2 \pm 3.8 days vs $4.9 \pm 2.4$ days, $P=.001$ ). Moreover, the length of hospital stay was significantly greater for patients with AF compared with patients who did not have AF both within the VATS cohort $(6.0 \pm 1.5$ days vs $4.7 \pm 2.5$ days, $P=.01)$ and the thoracotomy cohort $(9.2 \pm 4.3$ days vs 6.8 \pm 3.6 days, $P=.03$ ) (Table 3). Patients with AF were older than those without AF regardless of whether they had a VATS approach $(73 \pm 7$ years vs $66 \pm 9$ years, $P=.002)$ or a thoracotomy (72 \pm 7 years vs $66 \pm 10$ years, $P=$ $.005)$.

\section{Discussion}

The main finding of the present study is that postoperative AF after pulmonary lobectomy occurs with similar frequency whether a minimally invasive VATS or a traditional rib-spreading thoracotomy is performed. The overall rate of $\mathrm{AF}$ in all patients was $14 \%$, a figure consistent with that seen in previous series mentioned above, ${ }^{20-22}$ and after matching for age and gender there was no difference between patients who underwent VATS and patients who underwent thoracotomy. The precise cause of AF in these patients is still unclear, but the results suggest that autonomic denervation and stress-mediated neurohumoral mechanisms resulting from anatomic pulmonary resection, and not incision-related effects, are responsible for the pathogenesis of AF in susceptible individuals. ${ }^{1,20}$ As noted in prior studies, ${ }^{1-3,20-22}$ although $\mathrm{AF}$ frequently accompanies other comorbidities postoperatively, AF contributes to a longer hospitalization when compared with patients without AF undergoing the same operative approach.

Proponents of VATS for anatomic lung resection emphasize the benefits of decreased acute postoperative pain, shorter chest tube duration, and, consequently, shorter hospital stays. In addition, some authors have attempted to show that a less-invasive approach to lung resection results 
TABLE 3. Impact of atrial fibrillation on hospital stay

\begin{tabular}{|c|c|c|c|c|c|c|}
\hline & VATS/AF, $n=15$ & VATS/No AF, n = 107 & $P$ value & THOR/AF, $\mathbf{n}=\mathbf{2 0}$ & THOR/No AF, n = 102 & $P$ value \\
\hline Age (y) & $73 \pm 7$ & $66 \pm 9$ & .002 & $72 \pm 7$ & $66 \pm 10$ & .005 \\
\hline $\operatorname{LOS}(d)$ & $6.0 \pm 1.5$ & $4.7 \pm 2.5$ & .01 & $9.2 \pm 4.3$ & $6.8 \pm 3.6$ & .03 \\
\hline
\end{tabular}

LOS, Length of stay; VATS, video-assisted thoracic surgery; AF, atrial fibrillation; THOR, thoracotomy. Data are mean \pm standard deviation.

in fewer postoperative complications in high-risk patients, such as the elderly who might not otherwise tolerate thoracotomy. ${ }^{18,19}$ One of the most common postoperative complications after anatomic pulmonary resection is supraventricular arrhythmia, an event that results in an extended hospital stay with the attendant increased costs, as well as a greater risk of stroke. ${ }^{1,2}$ In several large retrospective studies that analyzed the incidence of and risk factors for postoperative AF after noncardiac thoracic surgical procedures, the observed rate was approximately $20 \% .^{20-22}$ The preferred surgical approach in the majority of these studies was thoracotomy. In contrast, Jaklitsch and colleagues $^{18}$ reported that the AF rate in 32 elderly patients undergoing VATS lobectomy was $3.1 \%$, whereas Gharagozloo and colleagues ${ }^{23}$ reported an arrhythmia rate of 9.4\% (17/179). McKenna and colleagues ${ }^{24}$ published the largest single institution series of 1100 VATS lobectomies and reported a postoperative AF rate of $2.9 \%$. These data suggest that a minimally invasive VATS technique, perhaps by obviating the surgical stress induced by a rib-spreading thoracotomy, may result in a decreased incidence of AF. The greater incidence of AF observed in our study may be explained by differences in AF definitions, monitoring techniques, and prevention strategies in each of the referenced studies. We recently observed that elderly patients undergoing a lobectomy have an incidence of AF of $27 \%$ when telemetry is used. ${ }^{25}$ Because of logistic constraints, our study used routine continuous telemetry in $23 \%$ of patients; as a result, we believe that our reported incidence of AF is actually an underestimate of the true rate and that we were unable to detect asymptomatic or "silent" episodes that required no intervention.

There were a few interesting trends when comparing the VATS and thoracotomy groups with regard to other aspects of their postoperative course. As expected and consistent with numerous previous reports, the length of hospital stay for patients undergoing VATS was significantly shorter than their thoracotomy counterparts by more than 2 days. This in part explains why the mean duration of hospitalization for patients undergoing VATS lobectomy who had AF was still shorter than that of patients undergoing thoracotomy without $\mathrm{AF}(6.0 \pm 1.5$ days vs $6.8 \pm 3.6$ days). The trend in the number and rate of overall and major pulmonary complications seemed to favor the VATS group, but this result did not reach statistical significance. Additional studies looking into this issue with a larger sample size might be useful.

\section{Strengths and Limitations}

There are several strengths of this study. First, we evaluated a common postoperative problem after anatomic lung resection in a homogeneous population of patients. Second, the definition and detection of the end point of $\mathrm{AF}$ was systematic, consistent, and clinically relevant. Third, by matching patients we attempted to eliminate selection bias as rigorously as possible outside of the setting of a randomized, prospective study. There are also several limitations. First, as mentioned above, telemetry was not routinely used in all patients once patients were discharged from the postanesthesia care unit. Second, despite matching, this is still a retrospective analysis, and there were inequalities between groups that were both known (use of induction therapy and preoperative carbon monoxide diffusion in the lung) and unknown. Third, it is possible that the sample size studied was not large enough to show that the $4 \%$ difference in AF rate was statistically significant. However, to discern a difference between a proportion of 0.12 and 0.16 in the binomial 2-sample case at an alpha of 0.5 and power of 0.8 , a study with 1180 patients in each group would be required. Last, although the results suggest that the operative approach is not a significant factor in the pathogenesis of postoperative $\mathrm{AF}$, the data offer little additional insight to further our understanding of mechanisms responsible for postoperative AF.

\section{Conclusions}

$\mathrm{AF}$ is a common postoperative complication in older patients undergoing anatomic pulmonary resection and contributes to prolonged hospitalization, associated health care costs, and the known risk for thromboembolic events. The use of a minimally invasive, non-rib spreading VATS approach does not decrease the incidence of postoperative AF when compared with standard thoracotomy. Patients deemed to be high risk preoperatively should be considered for proven prophylactic therapy regardless of the planned operative approach. ${ }^{25,26}$

\section{References}

1. Amar D. Perioperative atrial tachyarrhythmias. Anesthesiology. 2002; 97:1618-23. 
2. Polanczyk CA, Goldman L, Marcantonio ER, Orav EJ, Lee TH. Supraventricular arrhythmia in patients having noncardiac surgery: clinical correlates and effect on length of stay. Ann Intern Med. 1998;129:279-85.

3. Amar D, Zhang H, Leung DHY, Roistacher N, Kadish AH. Older age is the strongest predictor of postoperative atrial fibrillation. Anesthesiology.2002;96:352-6.

4. Gridelli C, Perrone F, Monfardini S. Lung cancer in the elderly. Eur J Cancer. 1997;33:2313-4.

5. Gridelli C. Chemotherapy of non-small cell lung cancer in the elderly. Lung Cancer. 2002;38(suppl 3):S67-S70.

6. Yancik R, Ries LA. Aging and cancer in America. Demographic and epidemiologic perspectives. Hematol Oncol Clin North Am. 2000;14: 17-23.

7. Lewis RJ. The role of video-assisted thoracic surgery for cancer of the lung: wedge resection to lobectomy by simultaneous stapling. Ann Thorac Surg. 1993;56:762-8.

8. Kirby TJ, Rice TW. Thoracoscopic lobectomy. Ann Thorac Surg. 1993;56:784-6.

9. Walker WS, Carnochan, FM, Pugh GC. Thoracoscopic pulmonary lobectomy. J Thorac Cardiovasc Surg.1993;106:1111-7.

10. McKenna RJ. Lobectomy by video-assisted thoracic surgery with mediastinal node sampling for lung cancer. J Thorac Cardiovasc Surg. 1994;107:879-82.

11. Landreneau RJ, Hazelrigg SR, Mack MJ, et al. Postoperative painrelated morbidity: video-assisted thoracic surgery versus thoracotomy. Ann Thorac Surg. 1993;56:1285-9.

12. McKenna RJ, Wolf RK, Brenner M, et al. Is lobectomy by videoassisted thoracic surgery an adequate cancer operation? Ann Thorac Surg. 1998;66:1903-8.

13. Kaseda S, Aoki T, Hangai N, et al. Better pulmonary function and prognosis with video-assisted thoracic surgery than with thoracotomy. Ann Thorac Surg. 2000;70:1644-6.

14. Sugi K, Kaneda Y, Esato K. Video-assisted thoracoscopic lobectomy achieves a satisfactory long-term prognosis in patients with clinical stage IA lung cancer. World J Surg. 2000;24:27-31.
15. Walker WS, Codispoti M, Soon SY, et al. Long-term outcomes following VATS lobectomy for non-small cell bronchogenic carcinoma. Eur J Cardiothorac Surg. 2003;23:397-402.

16. Thomas P, Doddoli C, Yena S, et al. VATS is an adequate oncological operation for stage I non-small cell lung cancer. Eur J Cardiothorac Surg. 2002;21:1094-9.

17. Daniels LJ, Balderson SS, Onaitis MW, et al. Thoracoscopic lobectomy: a safe and effective strategy for patients with stage I lung cancer. Ann Thorac Surg. 2002;74:860-4.

18. Jaklitsch MT, DeCamp MM Jr, Liptak MJ, et al. Video-assisted thoracic surgery in the elderly. Chest. 1996;110:751-8.

19. Demmy TL, Curtis JJ. Minimally invasive lobectomy directed toward frail and high-risk patients: a case-control study. Ann Thorac Surg. 1999;68:194-200.

20. Passman RS, Gingold DS, Amar D, et al. Prediction rule for atrial fibrillation after major noncardiac thoracic surgery. Ann Thorac Surg. 2005;79:1698-703.

21. Vaporciyan AA, Correa AM, Rice DC, et al. Risk factors associated with atrial fibrillation after noncardiac thoracic surgery: analysis of 2588 patients. J Thorac Cardiovasc Surg. 2004;127:779-86.

22. Roselli EE, Murthy SC, Rice TW, et al. Atrial fibrillation complicating lung cancer resection. J Thorac Cardiovasc Surg. 2005;130:438-44.

23. Gharagozloo F, Tempesta B, Margolis M, Alexander EP. Videoassisted thoracic surgery lobectomy for stage I lung cancer. Ann Thorac Surg. 2003;76:1009-15.

24. McKenna RJ Jr, Houck W, Fuller CB. Video-assisted thoracic surgery lobectomy: experience with 1,100 cases. Ann Thorac Surg. 2006;81: 421-6.

25. Amar D, Zhang H, Heerdt PM, Park B, Fleisher M, Thaler HT. Statin use is associated with a reduction in atrial fibrillation after noncardiac thoracic surgery independent of C-reactive protein. Chest. 2005;128: 3421-7.

26. Amar D, Roistacher N, Rusch VW, et al. Effects of diltiazem prophylaxis on the incidence and clinical outcome of atrial arrhythmias after thoracic surgery. J Thorac Cardiovasc Surg. 2000;120:790-8. 